\title{
Assistência ao parto de adolescentes e mulheres em idade materna avançada em maternidades vinculadas à Rede Cegonha
}

\author{
Childbirth care for adolescents and advanced maternal age \\ in maternities linked to Rede Cegonha
}

Elaine Fernandes Viellas (https://orcid.org/0000-0001-5259-8102) ${ }^{1}$

Thereza de Lamare Franco Netto (https://orcid.org/0000-0001-6186-8311) ${ }^{2}$

Silvana Granado Nogueira da Gama (https://orcid.org/0000-0002-9200-0387) ${ }^{1}$

Márcia Leonardi Baldisserotto (https://orcid.org/0000-0001-6907-2510) ${ }^{1}$

Priscila Fernandes do Prado Neto (https://orcid.org/0000-0001-9809-7186) ${ }^{3}$

Mariana Ramos Rodrigues (https://orcid.org/0000-0001-8493-1379) ${ }^{4}$

Katrini Guidolini Martinelli (https://orcid.org/0000-0003-0894-3241) ${ }^{5}$

Rosa Maria Soares Madeira Domingues (https://orcid.org/0000-0001-5722-8127) ${ }^{6}$

${ }^{1}$ Escola Nacional de Saúde Pública, Fiocruz. R. Leopoldo Bulhões 1480, Manguinhos. 21041-210 Rio de Janeiro RJ Brasil. elaine.viellas@gmail.com ${ }^{2}$ Secretaria de Estado da Justiça e Cidadania do Distrito Federal, Governo do Distrito Federal. Brasília DF Brasil.

${ }^{3}$ Desenvolvimento de Políticas Sociais, Ministério da Saúde. Brasília DF Brasil.

${ }^{4}$ Secretaria de Estado da Saúde do Distrito Federal, Governo do Distrito Federal. Brasília DF Brasil. ${ }^{5}$ Programa de PósGraduação em Saúde Coletiva, Universidade Federal do Espírito Santo. Vitória ES Brasil.

${ }^{6}$ Instituto Nacional de Infectologia Evandro Chagas, Fiocruz. Rio de Janeiro RJ Brasil.
Abstract Based on the Rede Cegonha guidelines that propose the strengthening of women's sexual and reproductive rights, we sought to present a brief overview of issues related to reproductive planning and to analyze obstetric practices in childbirth care for adolescents and women of advanced age in maternity hospitals linked to Rede Cegonha. Data were extracted from an assessment conducted in 2017, based on information from the interview with puerperal women and from the hospital record. For age extremes, the high proportion of unplanned pregnancies and low use of contraception means problems in accessing family planning programs. Adolescents are more exposed to the presence of a companion and less to the use of analgesia in labor. Advanced maternal age were more likely to use analgesia in labor and to give birth in the lithotomy position, being less exposed to amniotomy. Although Rede Cegonha is an excellent strategy for improving assistance to childbirth, attention is still needed to the use of potentially unnecessary or not recommended interventions, with greater incentive to good obstetric practices.

Key words Maternal age, Pregnancy, Maternal and child health services, Labor, Childbirth
Resumo Com base nas diretrizes da Rede Cegonha, que propõem fortalecimento dos direitos sexuais e dos direitos reprodutivos das mulheres, buscou-se apresentar um breve panorama de questões relacionadas ao planejamento reprodutivo $e$ analisar as práticas obstétricas na atenção ao parto de adolescentes e mulheres em idade avançada, em maternidades vinculadas a Rede Cegonha. Os dados foram extraídos da avaliação conduzida em 2017, com base nas informações da entrevista com as puérperas e do prontuário hospitalar. Para os extremos etários, a alta proporção de gravidez não planejada e a baixa utilização de meios de contracepção indicam problemas no acesso aos programas de planejamento familiar. As adolescentes estão mais expostas à presença de acompanhante e menos ao uso de analgesia no trabalho de parto. As mulheres em idade avançada apresentaram maior chance de uso de analgesia no trabalho de parto e de parir na posição de litotomia, estando menos expostas a prática de amniotomia. Apesar da Rede Cegonha ser uma excelente estratégia para melhoria da assistência ao trabalho de parto e ao parto, ainda é preciso atenção ao uso de intervenções potencialmente desnecessárias ou não recomendadas, com maior incentivo às boas práticas obstétricas.

Palavras-chave Idade materna, Gravidez, Serviços de saúde materno-infantil, Trabalho de parto, Parto 


\section{Introdução}

As Diretrizes Nacionais para Atenção Integral à Saúde de Adolescentes e Jovens ${ }^{1}$ seguem a definição da faixa etária da adolescência estabelecida pela Organização Mundial da Saúde (OMS)², que a delimita à segunda década de vida, ou seja, dos 10 aos 19 anos. Essas Diretrizes trazem os instrumentos legais de proteção do direito fundamental à saúde de adolescentes. O acesso universal e igualitário às ações e serviços para promoção, proteção e recuperação da saúde dessa população é garantido, por exemplo, pelo Estatuto da Criança e do Adolescente (ECA) ${ }^{3}$, que busca assegurar, inclusive, $\mathrm{o}$ atendimento pré e perinatal, somando duas prioridades para as políticas públicas: a adolescência e a gravidez. É o marco jurídico e legal que cria mecanismos para efetivação dos direitos fundamentais estabelecidos pela Constituição Federal, sob a concepção de crianças e adolescentes como sujeitos de direitos e como pessoas em desenvolvimento.

É ampla, porém não consensual, a literatura acerca da gravidez na adolescência. Estudos apontam maior frequência de baixo peso ao nascer e prematuridade em filhos de adolescentes, geralmente, justificando sua ocorrência pelas baixas condições socioeconômicas da maioria delas ${ }^{4}$, condições que se intensificam com as sucessivas gestações ainda nessa fase da vida ${ }^{5}$.

Mulheres que têm parto com 35 anos ou mais, são consideradas mulheres em idade materna avançada (IMA) e geralmente apresentam especificidades na saúde reprodutiva, estando mais propensas a desenvolver complicações na gestação e parto, principalmente, gestações de alto risco, como placenta prévia, descolamento prematuro de placenta e restrição do crescimento intrauterino $^{6}$. Entretanto, diferente do movimento observado para as mais jovens, não existem políticas públicas voltadas especificamente para o grupo de mulheres acima de 34 anos de idade, uma vez que os cuidados especializados se configuram dentro de diretrizes gerais e manuais do Ministério da Saúde (MS), como àqueles voltados para manejo da gestação de alto risco, do abortamento, do pré-natal e puerpério ${ }^{6,7}$, sem que haja políticas públicas voltadas especificamente para o grupo. Com o crescente número de mulheres que postergam a maternidade ${ }^{8}$, é de suma importância uma atenção obstétrica cuidadosa para minimizar possíveis danos à saúde da mulher e do bebê.

Visando estruturar e organizar a atenção à saúde materno-infantil no país, o MS instituiu, em 2011, a Rede Cegonha (RC), buscando asse- gurar a todas as mulheres, sejam adolescentes ou em idade avançada, o direito ao planejamento reprodutivo e a atenção humanizada à gravidez, ao parto e ao puerpério, bem como à criança o direito ao nascimento seguro e ao crescimento e desenvolvimento saudáveis9. Para isso, a estratégia RC vem priorizando ações relativas à adoção das boas práticas na assistência as parturientes nas maternidades, práticas essas recomendadas pela $\mathrm{OMS}^{10}$.

É nesse contexto de fortalecimento dos direitos sexuais e reprodutivos das mulheres, que o presente estudo buscou apresentar um breve panorama das questões relacionadas ao planejamento reprodutivo e analisar as práticas obstétricas na atenção ao parto de adolescentes e mulheres em idade avançada em maternidades vinculadas à Rede Cegonha, para subsidiar a oferta de atenção à saúde adequada às especificidades de cada um desses grupos etários.

\section{Método}

Este estudo é parte da "Avaliação da Atenção ao Parto e Nascimento em Maternidades da Rede Cegonha". Foi empregada a técnica de "Estimativa Rápida Participativa" - ERP ${ }^{11,12}$, recomendada pela Organização Pan-Americana de Saúde - OPS. A técnica consiste em uma abordagem simples e rápida para se obter informações que reflitam as condições locais, a partir da ótica dos diferentes atores sociais envolvidos com os problemas.

Todos os 606 hospitais públicos e mistos (privados conveniados ao SUS) que, em 2015, tinham plano de ação da RC participaram da avaliação. O volume de partos nesses hospitais representava quase $50 \%$ dos partos ocorridos naquele ano no país, segundo o Sistema de Informações sobre Nascidos Vivos - SINASC.

Foram consideradas elegíveis todas as mulheres que tiveram parto hospitalar no período do estudo, sendo excluídas apenas aquelas com transtorno mental grave, que não compreendiam o idioma português, surdas ou internadas na Unidade Intermediária ou Unidade de Terapia Intensiva no pós-parto. Todas as puérperas elegíveis internadas em cada unidade hospitalar no período de duração do trabalho de campo foram convidadas a participar do estudo. Informações mais detalhadas da metodologia do estudo estão disponíveis em Bittencourt et al. ${ }^{13}$.

A coleta de dados foi realizada entre dezembro de 2016 e outubro de 2017, tendo empregado cinco formulários eletrônicos específicos para 
cada fonte de dados. Utilizaram-se como métodos de coleta de dados: entrevista pessoal com informantes-chaves (gestores, profissionais de saúde e puérperas); extração de dados de prontuário, análise documental e observação in loco. Maiores detalhes são descritos em Vilella et al. ${ }^{14}$. Para a presente análise foram utilizadas informações obtidas por meio de entrevistas presenciais das mulheres após o parto e dados do prontuário hospitalar.

Três grupos de comparação foram criados para caracterizar as puérperas: adolescentes ( $\leq$ 19 anos), mulheres de 20 a 34 anos de idade, e mulheres em IMA ( $\geq 35$ anos).

Como covariáveis foram avaliadas: região de residência da mulher (Norte, Nordeste, Sul, Sudeste e Centro-oeste); cor da pele (segundo o Instituto Brasileiro de Geografia e Estatística: branca, preta, parda, amarela, indígena); escolaridade $(<$ ensino fundamental ou ensino médio e mais); situação conjugal (com ou sem companheiro); idade do pai do bebê (10-19 anos ou $\geq$ 20 anos); número de partos anteriores $(0,1,2, \geq$ $3)$; uso de método contraceptivo ( $\operatorname{sim}$ ou não); gravidez planejada (sim ou não); início precoce do pré-natal ( $1^{\circ}$ trimestre sim ou não); número de consultas de pré-natal $(0,1-3,4-6, \geq 7)$; tipo de parto (normal/fórceps ou cesariana). Todas as informações sobre as características sociodemográficas e reprodutivas da mulher foram extraídas do questionário de entrevista com a puérpera.

$\mathrm{Na}$ análise das práticas obstétricas durante o trabalho de parto e parto, foram avaliadas as boas práticas: oferta de líquidos e alimentos durante o trabalho de parto (dieta livre sim ou não); uso de métodos não farmacológicos para alívio da dor (sim ou não para uso de chuveiro/banheira com água quente, bola, massagem, cavalinho ou banquinho, posteriormente categorizada em sim e não); mobilidade durante o primeiro estágio do trabalho de parto (deambulação sim ou não); presença do acompanhante durante o período da hospitalização (em tempo integral ou parcial, posteriormente categorizada em sim e não). Foram também avaliadas as seguintes práticas prejudiciais ou que são usadas excessivamente: uso de cateter venoso; uso de ocitocina para aceleração do trabalho de parto; recebimento de analgesia raquidiana/epidural realização de amniotomia (ruptura artificial de membranas); posição de litotomia; manobra de Kristeller e episiotomia (corte perineal) - todas tendo como opção de resposta as categorias "sim e não".

Informações sobre alimentação durante o trabalho de parto, uso de métodos não farmaco- lógicos para alívio da dor, deambulação, presença de acompanhante, uso de cateter venoso, analgesia e manobra de Kristeller foram reportadas pelas puérperas na entrevista. As informações sobre uso de ocitocina, amniotomia, litotomia e episiotomia foram coletadas do prontuário hospitalar.

Para análise das práticas obstétricas durante o trabalho de parto e parto, as mulheres que não entraram em trabalho de parto e não tiveram parto vaginal foram excluídas. As características sociodemográficas e reprodutivas das mulheres, estratificadas por faixa etária materna, foram testadas através do teste do $\chi^{2}$. O nível de significância estabelecido foi de $20 \%$. Mesmo procedimento foi utilizado para comparar as proporções relativas à avaliação das práticas obstétricas no trabalho de parto e parto.

Modelos de regressão logística múltipla foram desenvolvidos para relacionar as práticas obstétricas no trabalho de parto e parto com a faixa etária da mulher. $\mathrm{O}$ nível de significância estabelecido foi nessa análise foi de 5\% e utilizaram-se as estimativas de razão de chances (OR) considerando o efeito do desenho amostral. As OR foram ajustadas para região geográfica, cor da pele, escolaridade e paridade, escolhidas através de critérios estatísticos e teóricos, selecionadas por representarem diferentes dimensões sociodemográficas com potencial para interferir na incidência das práticas obstétricas. Os softwares empregados nas análises foram o SPSS 20.0 e o Microsoft Excel versão 2007.

O estudo de avaliação foi aprovado pelo Comitê de Ética da Universidade Federal do Maranhão. A todas as mulheres foi lido o termo de consentimento livre e esclarecido (TCLE) e entregue uma cópia àquelas que concordaram em participar da avaliação. Foram adotados os devidos cuidados para garantir o sigilo e a confidencialidade dos dados.

\section{Resultado}

Foram avaliadas 606 maternidades vinculados à Rede Cegonha e entrevistadas 10.665 puérperas no pós-parto imediato. Do total de partos ocorridos, cerca de 20,5\% foram de adolescentes e $11 \%$ de mulheres em idade avançada. Dentre as adolescentes, menos de $1 \%$ tinham entre 12 e 14 anos de idade.

As adolescentes foram em maior proporção pardas e de menor escolaridade. Uma menor proporção delas referiu ter companheiro e cerca de 3/4 relataram que os pais dos bebês tinham 20 
anos e mais de idade (Tabela 1). Proporcional- 3 ou mais filhos. Menos da metade das mulheres mente, estão mais presentes nas regiões Norte e fizeram uso de algum método contraceptivo para Nordeste do país (Gráfico 1).

Em relação à saúde reprodutiva, destaca-se que mais de $80 \%$ das adolescentes eram nulíparas e $31 \%$ das mulheres em idade avançada tinham prevenir a gravidez atual. A gravidez foi planejada por $27 \%$ das adolescentes e $39 \%$ das mulheres em IMA. O pré-natal foi iniciado precocemente por $60 \%$ das adolescentes e $72 \%$ das mulheres de

Tabela 1. Características sociodemográficas e reprodutivas de puérperas segundo a faixa etária materna. Rede Cegonha - Brasil, 2017.

\begin{tabular}{|c|c|c|c|c|c|}
\hline \multirow{4}{*}{ Características } & \multicolumn{5}{|c|}{ Faixa etária } \\
\hline & $\leq 19$ anos & 20-34 anos & $\geq 35$ anos & Total & \multirow{3}{*}{ p valor } \\
\hline & $(n=2.192)$ & $(\mathrm{n}=7.302)$ & $(\mathrm{n}=1.169)$ & $(n=10.665)$ & \\
\hline & $\%$ & $\%$ & $\%$ & $\%$ & \\
\hline Todas as mulheres & 20,5 & 68,5 & 11,0 & - & - \\
\hline \multicolumn{6}{|l|}{ Cor da pele } \\
\hline branca & 23,5 & 26,1 & 29,5 & 26,0 & 0,092 \\
\hline preta & 13,4 & 13,3 & 13,6 & 13,3 & \\
\hline parda & 60,7 & 58,3 & 55,0 & 58,4 & \\
\hline amarelo/oriental & 1,8 & 1,6 & 1,4 & 1,6 & \\
\hline indígena & 0,6 & 0,7 & 0,5 & 0,7 & \\
\hline \multicolumn{6}{|l|}{ Escolaridade } \\
\hline ensino fundamental & 82,0 & 47,4 & 46,8 & 54,4 & $<0,001$ \\
\hline ensino médio e mais & 18,0 & 52,6 & 53,2 & 45,6 & \\
\hline \multicolumn{6}{|l|}{ Situação conjugal } \\
\hline sem companheiro & 31,4 & 19,2 & 17,5 & 21,5 & $<0,001$ \\
\hline com companheiro & 68,6 & 80,8 & 82,5 & 78,5 & \\
\hline \multicolumn{6}{|l|}{ Idade do pai do bebê } \\
\hline 10 a 19 anos & 27,4 & 4,3 & 2,7 & 8,9 & $<0,001$ \\
\hline 20 anos e mais & 72,6 & 95,7 & 97,3 & 91,1 & \\
\hline \multicolumn{6}{|l|}{ Número de partos anteriores } \\
\hline 0 & 81,6 & 41,2 & 22,9 & 47,5 & $<0,001$ \\
\hline 1 & 15,5 & 30,6 & 24,5 & 26,8 & \\
\hline 2 & 2,6 & 16,7 & 21,4 & 14,3 & \\
\hline$\geq 3$ & 0,3 & 11,5 & 31,2 & 11,4 & \\
\hline \multicolumn{6}{|l|}{ Uso de método contraceptivo } \\
\hline não & 63,8 & 52,3 & 53,2 & 54,8 & $<0,001$ \\
\hline $\operatorname{sim}$ & 36,2 & 47,7 & 46,8 & 45,2 & \\
\hline \multicolumn{6}{|l|}{ Gravidez planejada } \\
\hline não & 72,6 & 62,0 & 60,7 & 64,0 & $<0,001$ \\
\hline $\operatorname{sim}$ & 27,4 & 38,0 & 39,3 & 36,0 & \\
\hline \multicolumn{6}{|l|}{ Início precoce do pré-natal } \\
\hline não & 40,0 & 30,1 & 28,4 & 31,9 & $<0,001$ \\
\hline $\operatorname{sim}$ & 60,0 & 69,9 & 71,6 & 68,1 & \\
\hline \multicolumn{6}{|l|}{ No consultas de pré-natal } \\
\hline não fez pré-natal & 9,2 & 8,4 & 8,6 & 8,6 & $<0,001$ \\
\hline 1 a 3 consultas & 7,6 & 5,0 & 2,9 & 5,3 & \\
\hline 4 a 6 consultas & 27,3 & 23,1 & 21,3 & 23,8 & \\
\hline$>6$ consultas & 55,9 & 63,5 & 67,2 & 62,3 & \\
\hline \multicolumn{6}{|l|}{ Tipo de parto } \\
\hline normal/fórceps & 65,7 & 55,8 & 43,5 & 56,5 & $<0,001$ \\
\hline cesariana & 34,3 & 44,2 & 56,5 & 43,5 & \\
\hline
\end{tabular}




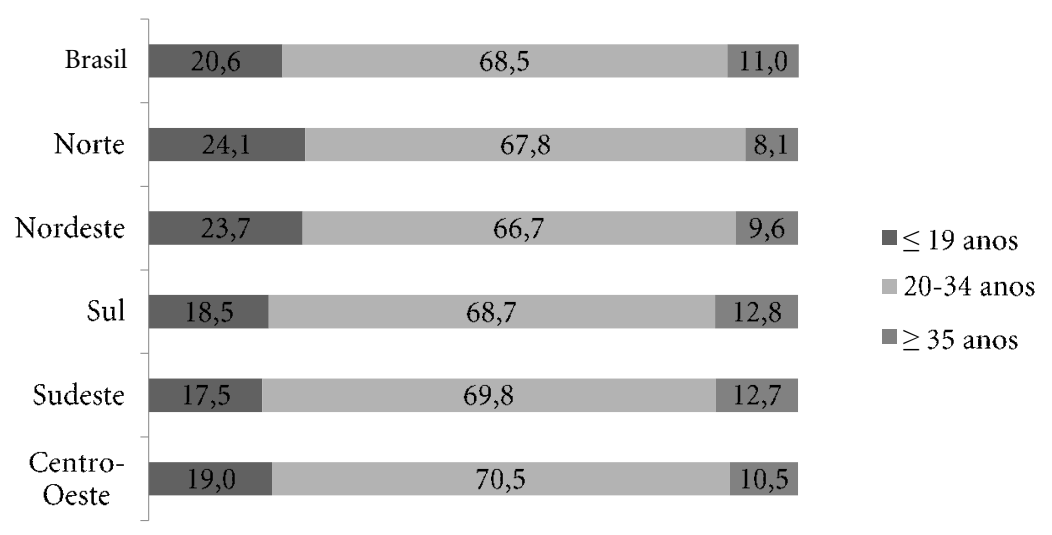

Gráfico 1. Distribuição das puérperas por macrorregião do país segundo os extremos etários. Rede Cegonha Brasil, 2017.

idade avançada. A maioria realizou mais de seis consultas de pré-natal, com proporção mais elevada no grupo de mulheres de 35 anos e mais de idade (Tabela 1). Nas maternidades da RC, mais da metade dos partos de todas as mulheres foi por via vaginal (normal ou fórceps), entretanto, a cesariana chegou a quase $60 \%$ no grupo de mulheres de idade avançada (Tabela 1).

Quanto às práticas obstétricas na atenção ao trabalho de parto e parto, dentre os métodos não farmacológicos para alívio da dor no trabalho de parto, destaca-se o uso de chuveiro ou banheira com água quente como técnica mais frequente $(47,4 \%)$. As adolescentes foram as que mais utilizaram os métodos não farmacológicos. Outras práticas favoráveis ao trabalho de parto como a livre movimentação e presença de acompanhante foram também mais frequentes no grupo das adolescentes (Tabela 2).

Ainda na Tabela 2, é possível observar que a amniotomia e uso da manobra de Kristeller foram menos frequentes nas mulheres com 35 anos e mais, sendo o uso de analgesia e a posição de litotomia para parir mais frequentes entre as mulheres dessa faixa etária.

Após o controle pelas variáveis de confundimento, e comparadas às mulheres de 20 a 34 anos, foi maior a chance de amniotomia e da presença do acompanhante entre as adolescentes, com menor exposição do grupo a analgesia no trabalho de parto. Para as mulheres em idade avançada foi maior a chance de uso de analgesia no trabalho de parto e menor a chance de realização de aminiotomia. Foi observada ainda dife- rença em relação posição para o parto, estando as mulheres em idade avançada mais expostas ao uso da posição litotômica para parir (Tabela 3).

\section{Discussão}

Lançada em 2011 com a proposta de qualificar os serviços ofertados pelo SUS', os resultados deste estudo mostram os avanços de algumas ações propostas pela Rede Cegonha, mas também as dificuldades para o alcance dos direitos básicos à vida sexual e reprodutiva das mulheres. Importantes disparidades sociodemográficas ainda persistem, diferenciando o perfil de mulheres em idade avançada daquelas que estão na adolescência.

A distribuição das mulheres por macrorregião evidenciou maior proporção de puérperas adolescentes nas regiões Norte e Nordeste, e de mulheres com 35 anos e mais no Sul e Sudeste do país. Segundo análise realizada pelo Instituto de Pesquisa Econômica Aplicada (IPEA) ${ }^{15}$ as regiões Norte e Nordeste concentram as cinco regiões metropolitanas do Brasil com maior Índice de Vulnerabilidade Social (IVS). Esse índice considera 16 indicadores, organizados em três dimensões. A dimensão infraestrutura urbana, que compreende os indicadores de coleta de lixo, água e esgoto inadequados e tempo de deslocamento casa-trabalho. A dimensão capital humano, que abarca os indicadores de mortalidade infantil, mães jovens (10 a 17 anos), mães sem o ensino fundamental e com filhos de até 15 anos de idade, analfabetismo, crianças em domicílios 
Tabela 2. Práticas obstétricas na atenção ao trabalho de parto e parto segundo faixa etária materna. Rede Cegonha - Brasil, 2017.

\begin{tabular}{|c|c|c|c|c|c|}
\hline \multirow[b]{2}{*}{ Prática obstétrica } & \multicolumn{5}{|c|}{ Faixa etária } \\
\hline & $\begin{array}{c}\leq 19 \text { anos } \\
(\%)\end{array}$ & $\begin{array}{c}20-34 \text { anos } \\
(\%)\end{array}$ & $\begin{array}{c}\geq 35 \text { anos } \\
(\%)\end{array}$ & Total (\%) & pvalor \\
\hline \multicolumn{6}{|l|}{ Chuveiro/banheira } \\
\hline uso & 52,1 & 46,7 & 40,1 & 47,4 & $<0,001$ \\
\hline \multicolumn{6}{|l|}{ Bola } \\
\hline uso & 33,7 & 27,1 & 22,1 & 28,2 & $<0,001$ \\
\hline \multicolumn{6}{|l|}{ Massagem } \\
\hline uso & 33,9 & 27,8 & 24,1 & 28,9 & $<0,001$ \\
\hline \multicolumn{6}{|l|}{ Cavalinho } \\
\hline uso & 12,2 & 10,2 & 7,8 & 10,5 & 0,029 \\
\hline \multicolumn{6}{|l|}{ Banquinho } \\
\hline uso & 13,5 & 12,6 & 7,9 & 12,4 & 0,015 \\
\hline \multicolumn{6}{|l|}{ Dieta livre } \\
\hline $\operatorname{sim}$ & 44,5 & 44,0 & 39,1 & 43,7 & 0,110 \\
\hline \multicolumn{6}{|l|}{ Deambulação } \\
\hline $\operatorname{sim}$ & 74,3 & 74,4 & 66,5 & 73,7 & 0,002 \\
\hline \multicolumn{6}{|l|}{ Acompanhante na internação } \\
\hline sim, mas não o tempo todo & 16,0 & 14,6 & 11,4 & 14,6 & $<0,001$ \\
\hline sim, o tempo todo & 76,5 & 73,6 & 70,6 & 74,0 & \\
\hline \multicolumn{6}{|l|}{ Cateter venoso $^{2}$} \\
\hline $\operatorname{sim}$ & 65,7 & 62,7 & 62,9 & 63,4 & 0,159 \\
\hline \multicolumn{6}{|l|}{ Ocitocina $^{1},^{2}$} \\
\hline $\operatorname{sim}$ & 35,4 & 36,1 & 34,1 & 35,8 & 0,614 \\
\hline \multicolumn{6}{|l|}{ Analgesia $^{2}$} \\
\hline $\operatorname{sim}$ & 18,3 & 19,9 & 23,2 & 19,8 & 0,031 \\
\hline \multicolumn{6}{|l|}{ Amniotomia $^{1},^{2}$} \\
\hline $\operatorname{sim}$ & 45,9 & 41,7 & 36,0 & 42,2 & $<0,001$ \\
\hline \multicolumn{6}{|l|}{ Litotomia $^{1}{ }^{3}$} \\
\hline $\operatorname{sim}$ & 86,2 & 87,0 & 91,7 & 87,2 & 0,034 \\
\hline \multicolumn{6}{|l|}{ Manobra de Kristeller ${ }^{3}$} \\
\hline $\operatorname{sim}$ & 21,3 & 14,8 & 12,9 & 16,2 & $<0,001$ \\
\hline \multicolumn{6}{|l|}{ Episiotomia $^{1},^{3}$} \\
\hline $\operatorname{sim}$ & 38,4 & 31,6 & 28,6 & 33,0 & $<0,001$ \\
\hline
\end{tabular}

em que ninguém tem o ensino fundamental completo, crianças de 0 a 5 anos fora da escola, crianças de 6 a 14 anos fora da escola e população que não estuda, não trabalha e tem baixa renda. E a dimensão renda e trabalho, a qual considera os indicadores de trabalho infantil, desocupação, ocupação informal sem ensino fundamental, baixa renda e dependente de idosos e renda menor ou igual a R\$255. A maior proporção de puérperas adolescentes em localidades de maior IVS reforça o entendimento de que a gravidez na adolescência está muitas vezes relacionada com a situação de vulnerabilidade social, conforme já destacado pelo Ministério da Saúde ${ }^{1}$.

Estudo nacional baseado em dados da pesquisa 'Nascer no Brasil'16 identificou que mesmo entre as mulheres com IMA existem diferenças nas características socioeconômicas, coexistindo as nulíparas e as multíparas de idade avançada. As primeiras, com nível socioeconômico mais elevado, têm perfil semelhante às mulheres de países desenvolvidos, com o adiamento da gestação ocorrendo em virtude da priorização dessas mulheres pela conclusão de um curso de nível 
Tabela 3. Associação das práticas obstétricas durante o trabalho de parto e parto e a faixa etária materna. Modelos multivariados com OR brutos e ajustados*. Rede Cegonha - Brasil, 2017.

\begin{tabular}{lcccccc}
\hline \multirow{2}{*}{ Prática obstétrica } & \multicolumn{7}{c}{ Faixa etária } \\
\cline { 2 - 7 } & \multicolumn{3}{c}{$\leq \mathbf{1 9}$ anos } & \multicolumn{3}{c}{$\geq \mathbf{3 5}$ anos } \\
\cline { 2 - 7 } & OR(br) & OR(aj) & IC & OR(br) & OR(aj) & IC \\
\hline Práticas obstétricas no trabalho de parto & & & & & & \\
Uso de MNF para alívio da dor & 1,22 & 1,20 & $0,97-1,50$ & 0,77 & 1,01 & $0,78-1,31$ \\
Dieta livre & 1,02 & 1,00 & $0,81-1,22$ & 0,82 & 0,86 & $0,66-1,11$ \\
Deambulação & 1,00 & 0,86 & $0,68-1,08$ & 0,68 & 0,84 & $0,65-1,10$ \\
Presença de acompanhante & 1,67 & 1,30 & $1,01-1,73$ & 0,61 & 0,88 & $0,64-1,20$ \\
Cateter venoso & 1,14 & 1,00 & $0,84-1,22$ & 1,01 & 0,97 & $0,76-1,25$ \\
Analgesia & 0,90 & 0,76 & $0,60-0,94$ & 1,22 & 1,25 & $1,02-1,67$ \\
Amniotomia & 1,19 & 1,22 & $1,01-1,46$ & 0,79 & 0,69 & $0,54-0,88$ \\
Práticas obstétricas no parto & & & & & & \\
Litotomia & 0,93 & 0,92 & $0,70-1,20$ & 1,64 & 1,61 & $1,05-2,46$ \\
Manobra de Kristeller & 1,56 & 1,18 & $0,93-1,49$ & 0,85 & 0,88 & $0,61-1,30$ \\
Episiotomia & 1,35 & 0,90 & $0,73-1,10$ & 0,86 & 1,33 & $0,99-1,80$ \\
\hline
\end{tabular}

${ }^{*}$ Modelos logísticos ajustados pelas variáveis: região, cor da pele, escolaridade e paridade. MNF: método não farmacológico. IC: intervalo de $95 \%$ de confiança. OR: Odds Ratio ; br = bruta; aj = ajustada.

superior e de uma carreira profissional, antes de planejar a maternidade. As multíparas, por sua vez, pertencem a famílias de mais baixa renda e proles numerosas, que ainda se reproduzem nessa idade por falta de planejamento familiar.

Tendo apenas $27 \%$ das adolescentes e 39\% das mulheres em idade avançada planejado a gravidez atual e sendo baixa a utilização de meios de contracepção, os problemas no acesso aos programas de planejamento familiar ficaram evidentes nesse contexto. O planejamento reprodutivo envolve atividades educativas para levar informação, ofertando conhecimentos necessários para a escolha e a utilização de medidas contraceptivas adequadas, propiciando questionamentos e reflexões sobre temas relacionados com a prática da anticoncepção. Em um extremo, oportunidades estão sendo perdidas para aconselhamento dos adolescentes sobre saúde sexual e reprodutiva, com garantia de acesso e orientações para uso regular de métodos contraceptivos e prevenção de doenças sexualmente transmissíveis ${ }^{17}$. No outro, pouca atenção à realidade diferenciada das gestações tardias, em que as mulheres optam por postergar a maternidade para investir primeiro na formação, carreira e inserção no mercado de trabalho ${ }^{18,19}$, dando menor atenção, mesmo que não intencional, ao aumento das comorbidades e riscos obstétricos que estão associados à idade avançada ${ }^{20}$.

As ações voltadas ao planejamento reprodutivo no SUS não estão sendo suficientes para que homens e mulheres façam escolhas contraceptivas seguras e adequadas que lhes permitam decidir em que momento ter ou não ter filhos. Há que se destacar que durante a implementação da RC houve um importante avanço em relação a entrada dos homens nos serviços de saúde, com o "Pré-natal do Parceiro". Uma estratégia para exercício consciente da paternidade, com inclusão e participação ativa dos pais ou parceiros nos cuidados na gestação e parto ${ }^{21}$.

A atenção primária deve ser melhor aproveitada como o local mais apropriado de acesso aos métodos contraceptivos, ao programa de planejamento reprodutivo, às informações e aos cuidados com a saúde da mulher sem juízo de valor ou preconceitos $^{22}$, com garantia de acesso facilitado à contracepção de emergência, e de divulgação e incentivo ao uso de preservativo feminino ${ }^{23}$. No estudo, observamos que nenhuma mulher em IMA e menos de $1 \%$ das adolescentes que estavam utilizando algum método contraceptivo, referiram a escolha do preservativo feminino para evitar a gravidez atual (dado não mostrado em tabela).

Identificado em estudos anteriores ${ }^{24,25}$, o prénatal, uma ação programática típica da atenção primária, também enfrenta dificuldades para fornecer uma assistência de qualidade, com precariedade no fornecimento de orientações sobre práticas benéficas para facilitar o trabalho de parto e parto, de grande relevância para a promoção do parto vaginal. 
Entretanto, no presente estudo, foi possível observar que as adolescentes parecem se apropriar melhor das práticas obstétricas benéficas ao trabalho de parto e parto. Elas representam o grupo que mais utilizou métodos não farmacológicos para alívio da dor, que mais deambulou e contou com a presença do acompanhante, o que pode representar um avanço na atenção ao parto vaginal de adolescentes nas maternidades vinculadas à Rede Cegonha.

As adolescentes apresentaram $30 \%$ a mais de chance de ter um acompanhante na internação para o parto do que mulheres de 20 a 34 anos. A Lei Federal no 11.108 , de 2005, regulariza a presença do acompanhante de livre escolha da mulher durante o trabalho parto, parto e puerpério ${ }^{26}$. Talvez por ser um direito também assegurado pelo Estatuto da Criança e Adolescente (ECA) $)^{3}$, reforça a garantia de um acompanhante à adolescente, no caso identificado como seu responsável legal.

Desconsiderando a idade materna, mas ainda em relação ao cumprimento da lei que garante à gestante e parturiente o direito ao acompanhante, cabe destacar que mais de $70 \%$ das puérperas tiveram acompanhante em tempo integral ao longo da internação e quase $15 \%$ de forma parcial. Há alguns anos, o estudo 'Nascer no Brasil' ${ }^{27}$ identificou que menos de $20 \%$ das mulheres tiveram acompanhante de forma contínua e $57 \%$ parcialmente.

Cuidar da gestante e puérpera adolescente significa proporcionar atenção integral sensível às especificidades dessa faixa etária, com ações de promoção do autocuidado, de planejamento reprodutivo (para que a gravidez ocorra no momento da vida escolhido, podendo ser durante a adolescência ou não), com atenção aos aspectos éticos e legais do atendimento e direitos sexuais dos adolescentes. A mãe adolescente precisa estar melhor informada e precisa ser ouvida e atendida em seus direitos, tendo o pai adolescente o direito de ser seu acompanhante.

A Rede Cegonha se apresenta como uma oportunidade extraordinária para o avanço dessas questões, particularmente quanto ao uso de métodos anticoncepcionais e ao planejamento de ações educativas que promovam o vínculo dos adolescentes às unidades de saúde $\mathrm{e} o$ direito ao parto humanizado ${ }^{28}$. É preciso compreender melhor as normas para evitar interpretações equivocadas ou subjetivas que possam restringir o direito à saúde desta população só pelo fato de serem adolescentes $^{29}$.

Em particular para as mulheres em idade avançada, o planejamento familiar é fundamen- tal para melhoria da assistência já que os riscos de complicações aumentam com a idade e a contracepção é uma forma de preveni-los e de reduzir a mortalidade materna ${ }^{6,30}$. É preciso reconhecer o perfil diferenciado dessas mulheres, fornecendo aconselhamento adequado sobre métodos contraceptivos seguros, para que possam escolher o que melhor se adeque ao seu perfil e garanta a decisão sobre o melhor momento para engravidar.

A abordagem eficaz também será capaz de elucidar problemas relacionados à gravidez tardia e prevenir riscos, principalmente, àquelas de menor renda e grandes multíparas. $\mathrm{O}$ processo deve ser continuado durante o pré-natal, incentivando mulheres com 35 anos e mais à adoção das boas práticas, demonstrando seus benefícios para promoção do parto vaginal. É possível que os métodos não farmacológicos para alívio da dor estejam sendo pouco valorizados e subutilizados pelas mulheres em idade avançada nas maternidades da Rede Cegonha uma vez que a maior adoção dos métodos pelas adolescentes mostra também uma relação da idade com a menor demanda pela analgesia.

A oferta de analgesia medicamentosa é disponibilizada para mulheres durante o trabalho de parto, para garantir um parto sem dor $^{31}$. No presente estudo, as mulheres em IMA tiveram chance $25 \%$ maior de receber analgesia no trabalho de parto do que mulheres de 20 a 34 anos, corroborando com outros estudos que encontraram maior recebimento de anestesia peridural ou raquidiana em partos vaginais de mulheres em idade avançada ${ }^{32,33}$. Uma possível explicação pode ser em razão da maior proporção dessas mulheres já terem experimentado partos anteriores e, portanto, já entenderem como se comportar e o que solicitar. Ou outra explicação pode ser em função da maior utilização da posição de litotomia e menor movimentação durante trabalho de parto observado nesse grupo de mulheres, o que pode aumentar a dor e, consequentemente, o pedido pelo recurso farmacológico.

Outra técnica utilizada em obstétrica é a realização de amniotomia, que tem sido usada de forma rotineira nas maternidades para potencializar a aceleração do trabalho de parto, aumentando as contrações uterinas ${ }^{34,35}$. Segundo Santos et al. ${ }^{36}$, a técnica tem sido utilizada inclusive com maior frequência nas adolescentes de forma proposital, ocorrendo pela intenção do profissional em "facilitar" o processo para as adolescentes, tentando reduzir a duração do seu trabalho de parto. Apesar de bastante difundida, é preciso ter cautela pois dependendo da situação seu uso 
pode ser prejudicial, levando a desacelerações precoces dos batimentos cardíacos fetais e maior o risco de infecção ovular e puerperal ${ }^{37}$.

No que diz respeito à liberdade de posição e movimentação, a recomendação do MS é que os profissionais de saúde incentivem as parturientes a adotarem posições não supinas no trabalho de parto e parto para redução da sensação dolorosa na fase expulsiva ${ }^{33}$. No entanto, mesmo as evidências científicas demonstrando estes e outros benefícios $^{38}$, quase $90 \%$ das mulheres desse estudo utilizaram essa posição na hora do parto e as mulheres com 35 anos e mais foram as que mais deram à luz nessa clássica posição de litotomia. Estudos na área mostram que o uso da posição de litotomia pode estar muito relacionada às práticas tradicionalistas, que não se utilizam de outras possíveis posições para ajudar a mulher no parto ${ }^{33}$.

A episiotomia, por sua vez, é uma técnica que vem sendo utilizada com a justificativa de ampliação do canal do parto na intenção de reduzir uma possível laceração natural do períneo ${ }^{36,39}$. O que as evidências atuais demonstram não ser verdadeiro. $\mathrm{O}$ uso rotineiro não está associado a menos lesões do que seu uso restrito ${ }^{36}$. Sua indicação não tem sido criteriosa, e desconsidera os prováveis desfechos que pode ocasionar: hemorragia, dor no pós-parto, edema, infecções, problemas de cicatrização, hematoma e fístulas retovaginais, por exemplo ${ }^{40,41}$. A recomendação da OMS, em seu manual mais recente, não é de proibir a episiotomia, mas de restringir seu uso ${ }^{42}$.

$O$ estudo não encontrou associação estatisticamente significativa entre a idade materna avançada e a realização de episiotomia conforme verificado por outros estudos na área ${ }^{43,44}$. De forma geral, a associação é justificada pela maior proporção de gravidez de alto risco no grupo, seja pela diminuição na elasticidade do tecido com o aumento da idade materna, necessitando do corte para facilitar o parto ${ }^{44}$; seja para evitar complicações durante o parto dessas mulheres ${ }^{45}$; ou mesmo pela relutância dos profissionais em abandonar o velho hábito, conforme afirmam Wu et al. ${ }^{46}$.

Seja na adolescência ou em idade avançada, ainda é possível observar o uso de alguns procedimentos dolorosos, com baixo incentivo às práticas que facilitariam o parto das mulheres. Para Sousa et al.$^{47}$, todas essas práticas utilizadas para aceleração do trabalho de parto e parto deveriam ser abolidas das práticas rotineiras em obstetrícia uma vez que são prejudiciais ao processo espontâneo, causando sérios danos à saúde da mulher e do bebê.
A permanência do uso de algumas práticas intervencionistas reforça a necessidade de incentivo à melhoria da assistência prestada à mulher no trabalho de parto e parto nas maternidades vinculadas a Rede Cegonha. Os profissionais ainda precisam ser devidamente qualificados, trabalhando mais com protocolos e diretrizes. De acordo com Canto et al. ${ }^{48}$, a maternidade em idade avançada não é uma contraindicação, mas é preciso vigilância às vulnerabilidades clínico-obstétricas do grupo, buscando atender aos princípios da Política Nacional de Humanização da Atenção, garantindo acolhimento e classificação de risco em tempo oportuno pelos serviços de saúde.

Diante do aumento do número de mulheres que vêm postergando a maternidade, é importante criar um protocolo de atendimento voltado para essas mulheres de idade materna avançada. Seria um guia para os profissionais de saúde que atuam no SUS sobre ações voltadas para mulheres que engravidam tardiamente, em que se destaque o maior risco de desfechos negativos e os cuidados que precisam ser tomados para diminuir os riscos associados ao aumento da idade materna.

Considerando as especificidades das adolescentes, conclui-se que é importante incentivar cada vez mais a adoção do uso de práticas baseadas em evidencias, para reforçar e garantir a continuidade do cuidado, sem esquecer das ações de promoção do autocuidado, planejamento reprodutivo e atenção aos direitos sexuais e reprodutivos das adolescentes, descritos anteriormente. Ao engravidar, sendo de forma planejada ou não, os projetos de vida das adolescentes são em geral alterados, contribuindo na maioria das vezes para o abandono escolar, aumento das desigualdades e exclusão social².

A gestão também pode e deve exercer seu papel sobre o modelo de atenção obstétrica adotado nas maternidades uma vez que é o norteador das práticas assistenciais desenvolvidas nos serviços de saúde. Ao garantir o seguimento das práticas obstétricas úteis, com redução ou eliminação daquelas frequentemente utilizadas de modo inadequado ou danoso, poderá a gestão contribuir para a melhoria da assistência para todas as mulheres, em qualquer faixa etária.

É claro que há de se considerar as potencialidades do serviço para atender a clientela de forma resolutiva. A Rede Cegonha tem grande parte de suas ações voltadas ao funcionamento do sistema de cuidado, que incluem a disponibilidade de equipamentos, de instrumentos cirúrgicos, de 
Unidades de Terapia Intensiva, recursos financeiros e capacitação de recursos humanos, por exemplo ${ }^{9,10}$.

Sendo a Rede Cegonha uma estratégia útil de gestão, monitoramento e avaliação dos cuidados com as gestantes, puérperas e seus bebês, e mesmo que ainda precise de aprimoramento, claramente representa uma excelente oportunidade de melhoria da atenção integral à saúde das mulheres adolescentes e em idade materna avançada.

\section{Colaboradores}

EF Villelas, TDL Franco Netto e SGN Gama trabalharam na concepção e metodologia do artigo, na análise e interpretação dos dados, e redação do texto. PF Prado Neto, MR Rodrigues, ML Baldisserotto, KG Martinelli, RMSM Domingues contribuíram para a redação e realizaram a revisão crítica do texto. Todos os autores aprovaram a versão final do artigo. 


\section{Referências}

1. Brasil. Ministério da Saúde (MS). Diretrizes Nacionais para Atenção Integral à Saúde de Adolescentes e Jovens na Promoção, Proteção e Recuperação da Saúde. Brasília: MS; 2010. (Série A. Normas e Manuais Técnicos).

2. World Health Organization (WHO). Young People's Health - a Challenge for Society. Report of a WHO Study Group on Young People and Health for All. Geneva: WHO; 1986. (Technical Report Series 731).

3. Brasil. Lei $n^{\circ} 8.069$, de 13 de julho de 1990. Dispõe sobre o Estatuto da Criança e do Adolescente e dá outras providências. Diário Oficial da União 1990; 16 jul.

4. Amjad S, MacDonald I, Chambers T, Osornio Vargas A, Chandra S, Voaklander D, Ospina MB. Social determinants of health and adverse maternal and birth outcomes in adolescent pregnancies: A systematic review and meta analysis. Paediatr Perinat Epidemiol 2019; 33(1):88-99.

5. Silva AAA, Coutinho IC, Katz L, Souza ASR. Fatores associados à recorrência da gravidez na adolescência em uma maternidade escola: estudo caso-controle. Cad Saude Publica 2013; 29(3):496-506.

6. Brasil. Ministério da Saúde; Secretaria de Atenção à Saúde, Departamento de Ações Programáticas Estratégicas. Gestação de alto risco: manual técnico. Brasília: MS; 2012. 302 p. - (Série A. Normas e Manuais Técnicos).

7. Brasil. Ministério da Saúde (MS). Protocolos da Atenção Básica: Saúde das Mulheres. Brasília: MS, Instituto Sírio-Libanês de Ensino e Pesquisa; 2016.

8. Brasil. Ministério da Saúde (MS). Sistema de Informação de Nascidos Vivos. Datasus; 2019. [acessado 2019 Out 5]. Disponível em: http://tabnet.datasus.gov.br/ cgi/tabcgi.exe?sinasc/cnv/nvuf.def

9. Brasil. Ministério da Saúde (MS). Portaria no 1.459, de 24 de junho de 2011. Institui, no âmbito do SUS, a Rede Cegonha. Diário Oficial da União 2011; 24 jun.

10. World Health Organization (WHO). Care in normal birth: a practical guide. Geneva: WHO; 1996.

11. Piovesan MF, Padrão MVV, Dumont MU, Gondim GM, Flores O, Pedrosas JI, Lima LFM. Vigilância Sanitária: uma proposta de análise dos contextos locais. Rev. Bras. Epidemiol 2005; 8(1):83-95.

12. Carneiro FF, Hoefel MG, Silva MAM, Nepomuceno AR, Vilela C, Amaral FR, Carvalho GPM, Batista JL, Lopes PA. Mapeamento de vulnerabilidades socioambientais e de contextos de promoção da saúde ambiental na comunidade rural do Lamarão, Distrito Federal, 2011. Rev. bras. Saúde oсир 2012; 37(125):143-148.

13. Bittencourt SDA, Vilela MEA, Marques CO. Atenção ao Parto e Nascimento em Maternidades da Rede Cegonha: avaliação do grau de implantação das ações. Cien Saude Coletiva 2020; 26(3):801-822.

14. Vilela MEA, Leal MC, Thomaz EBAF, Gomes MASM, Bittencourt SDA, Gama SGN, Silva LBRAA, Lamy ZC. Avaliação da atenção ao parto e nascimento nas maternidades da Rede Cegonha: os caminhos metodológicos. Cien Saude Coletiva 2020; 26(3):789-800.

15. Costa MA, Marguti BO. Atlas da vulnerabilidade social nas regiões metropolitanas brasileiras. Brasília: IPEA; 2015. [acessado 2019 Out 15]. Disponível em: http:// ivs.ipea.gov.br/images/publicacoes/Ivs/publicacao_ atlas_ivs_rm.pdf
16. Martinelli KG. Implicações da idade materna avançada em desfechos maternos e perinatais [tese]. Rio de Janeiro: Fiocruz; 2018.

17. Brasil. Ministério da Saúde (MS). Cuidando de Adolescentes: orientações básicas para a saúde sexual e a saúde reprodutiva. Brasília: MS; 2015.

18. Baldwin MK, Jensen JT. Contraception during the perimenopause. Maturitas 2013; 76(3):235-242.

19. Lampinen R, Vehviläinen-Julkunen K, Kankkunen P. A review of pregnancy in women over 35 years of age. Open Nurs J 2009; 3:33-38.

20. Guedes MM. Nascimento do primeiro filho em idade materna avançada: percursos conducentes à sua ocorrência e adaptação dos casais nos primeiros seis meses de vida do bebê [tese]. Coimbra: Universidade de Coimbra; 2014.

21. Brasil. Ministério da Saúde (MS). Guia do Pré-Natal do Parceiro para Profissionais de Saúde. Brasília: MS; 2016.

22. Brasil. Ministério da Saúde (MS). Cadernos HumanizaSUS. Formação e intervenção. Brasília: MS; 2010. (Série B. Textos Básicos de Saúde).

23. Figueiredo R, Castro Filho JM, Kalckmann S. Os desafios do trabalho na atenção básica - Planejamento Familiar e Reprodutivo na Atenção Básica do Município de São Paulo: direito constitucional respeitado? Boletim do Instituto de Saúde 2014; 15(2):81-93.

24. Viellas EF, Domingues RMSM, Dias MAB, Gama SGN, Theme Filha MM, Costa JV, Bastos MH, Leal MC. Assistência pré-natal no Brasil. Cad Saude Publica 2014; 30(Supl.):S85-S100.

25. Domingues RMSM, Viellas EF, Dias MAB, Torres JA, Theme-Filha MM, Gama SGN, Leal MC. Adequação da assistência pré-natal segundo as características maternas no Brasil. Rev Panam Salud Publica 2015 37(3):140-147.

26. Brasil. Lei $\mathrm{n}^{\circ} 11.108$, de 7 de abril de 2005. Altera a Lei no 8.080, de 19 de setembro de 1990, para garantir às parturientes o direito à presença de acompanhante durante o trabalho de parto, parto e pós-parto imediato, no âmbito do Sistema Único de Saúde - SUS. Diário Oficial da União 2005; 7 abr.

27. Diniz CSG, d'Orsi E, Domingues RMSM, Torres JA, Dias MAB, Schneck CA, Lansky S, Teixeira NZF, Rance S, Sandall J. Implementação da presença de acompanhantes durante a internação para o parto: dados da pesquisa Nascer no Brasil. Cad Saude Publica 2014; 30(Supl.):S140-S153.

28. Lamare T. Cuidando de adolescentes na Rede Cegonha. Adolesc Saude 2013; 10(Supl. 1):6.

29. Brasil. Ministério da Saúde (MS). Marco legal: saúde, um direito de adolescentes. Brasília: MS; 2005. (Série A. Normas e Manuais Técnicos).

30. Brasil. Ministério da Saúde (MS). Política nacional de atenção integral à saúde da mulher: princípios e diretrizes. Brasília: MS; 2005. (C. Projetos, Programas e Relatório).

31. Gambling D, Berkowitz J, Farrell TR, Pue A, Shay D. A randomized controlled comparison of epidural analgesia and combined spinal-epidural analgesia in a private practice setting: pain scores during first and second stages of labor and at delivery. Anesth Analg 2013; 116(3):636-643. 
32. Schildberger B, Linzner D, Hehenberger L, Leitner $\mathrm{H}$, Pfeifer C. Influence of Maternal Age on Selected Obstetric Parameters. Geburtshilfe Frauenheilkd 2019; 79(11):1208-1215.

33. Bernis C, Varea C. Hour of birth and birth assistance: from a primate to a medicalized pattern? Am J Hum Biol 2012; 24(1):14-21.

34. Vogt SE, Diniz SG, Tavares CM, Santos NCP, Schneck CA, Zorzam B, Vieira DA, Silva KS, Dias MAB. Características da assistência ao trabalho de parto e parto em três modelos de atenção no SUS, no Município de Belo Horizonte, Minas Gerais, Brasil. Cad Saude Publica 2011; 27(9):1789-1800.

35. Leal MC, Pereira APE, Domingues RMSM, Theme Filha MM, Dias MAB, Nakamura-Pereira M, Bastos MH, Gama SGN. Intervenções obstétricas durante o trabalho de parto e parto em mulheres brasileiras de risco habitual. Cad Saude Publica 2014; 30(Supl.):S17-S47.

36. Santos NCP, Vogt SE, Pimenta AM, Duarte ED, Madeira LM, Abreu MNS, Léon RGP. Resultados maternos e neonatais no trabalho de parto e parto de adolescentes admitidas em um Centro de Parto Normal brasileiro. Adolesc Saude 2014; 11(3):39-50.

37. Brasil. Ministério da Saúde (MS). Parto, aborto e puerpério: assistência humanizada à mulher. Brasília: MS; 2001.

38. Previatti JF, Souza KV. Episiotomia: em foco a visão das mulheres. Rev Bras Enferm 2007; 60(2):197-201.

39. Corrêa Junior MD, Passini Júnior R. Selective Episiotomy: Indications, Techinique, and Association with Severe Perineal Lacerations. Rev Bras Ginecol Obstet 2016; 38(6):301-307.

40. Diniz SG, Chacham AS. O "corte por cima" e o "corte por baixo": o abuso de cesáreas e episiotomias em São Paulo. Questões de Saúde Reprodutiva 2006; I(1):8091.

41. Santos JO, Shimo AKK. Prática rotineira da episiotomia refletindo a desigualdade de poder entre profissionais de saúde e mulheres. Esc Anna Nery Rev Enferm 2008; 12(4):645-650.

42. World Health Organization (WHO). Managing complications in pregnancy and childbirth: a guide for midwives and doctors. $2^{\text {nd }}$ ed. Geneva: WHO; 2017. [acessado 2020 Jul 19]. Disponível em: https://www. who.int/maternal_child_adolescent/documents/managing-complications-pregnancy-childbirth/en/
43. Brasil. Ministério da Saúde (MS). Parto, aborto e puerpério: assistência humanizada à mulher. Brasília: MS; 2001.

44. Kaddoura R, DeJong J, Zurayk H, Kabakian T, Abbvad C, Mirza FG. Episiotomy practice in the Middle East: a Lebanese teaching tertiary care centre experience. Women Birth 2019; 32(2):223-228.

45. Carrolli G, Mignini L. Episiotomy for vaginal birth. Cochrane Database Syst Rev 2009; (1):CD000081.

46. Wu LC, Malhotra R, Allen Junior JC, Lie D, Tan TC, Ostbye T. Risk factors and midwife-reported reasons for episiotomy in women undergoing normal vaginal delivery. Arch Gynecol Obstet 2013; 288(6):1249-1256.

47. Sousa AMM, Souza KV, Resende EM, Martins EF, Campos D, Lansky S. Práticas na assistência ao parto em maternidades com inserção de enfermeiras obstétricas, em Belo Horizonte, Minas Gerais. Esc Anna Nery 2016; 20(2):324-331.

48. Canto MJ, Reus A, Cortés S, Ojeda F. Pregnancy outcome in a Spanish population of women beyond age 40 delivered above 32 weeks' gestation. J Matern Fetal Neonatal Med 2012; 25(5):461-466.

Artigo apresentado em 30/04/2020

Aprovado em 27/07/2020

Versão final apresentada em 29/07/2020

Editores-chefes: Romeu Gomes, Antônio Augusto Moura da Silva 